\title{
GENETIC ASPECTS OF MALARIA PARASITE INFECTION AND THE HOST IMMUNE RESPONSE IN RELATION TO PARASITE EVASION
}

\author{
L. PEREIRA DA SILVA
}

\section{SuMmary}

In relation to problems found upto now for the development of vaccines against malaria, recent studies have pointed out the different strategies evolved by the parasites to evade the host immune response. They include antigenic variation, antigenic polymorphism, antigenic mimicry and direct interference with the gene- ration of the host immune response. These different mechanisms are rapidly reviewed and discussed with the aim of foccusing the actual orientation of basic and applied research which has been developed to overcome these difficulties.

RÉSumÉ : Aspects génétiques de l’infection paludéenne et de la réponse immunitaire de l'hôte en rapport avec l'échappement du parasite.

Pour comprendre les problèmes rencontrés jusqu'à présent pour le développement de vaccins anti-paludéens, de nombreuses recherches ont été entreprises récemment sur les stratégies créées par le parasite pour échapper à la réponse de l'hôte. Elles comprennent la variation antigénique, le polymorphisme anti- génique, le mimétisme antigénique et l'interférence directe avec la réponse immunitaire de l'hôte. Ces différents mécanismes sont discutés dans le but de définir l'orientation actuelle des recherches visant à dépasser ces difficultés.
Malarial parasites have evolved different strategies to evade the host's immune response. These represent major problems in the rational development of malaria vaccines. An efficient mechanism developed by the parasite to survive the host's response is to invade liver cells where, in the case of Plasmodium vivax, parasites can remain dormant for long periods. After leaving the hepatic cells and invading erythrocytes parasites can also efficiently survive, even after the development of a protective immune response. This is able to destroy most parasites, but is usually unable to establish sterile immunity. Plasmodium malariae, in particular, is known for its ability to remain an asymtomatic erythrocytic infection for many years. Plasmodium falciparum has developed a different strategy. It provokes serious diseases in the first few infections in children or unprotected adults, but afterwards it induces a non sterile protective immunity. This, however, rapidly disappears when the contact with the parasite is interrupted (curing the infection by chemotherapy or leaving the transmission area). People living in the endemic area are, nevertheless, continuously reinfected and even when harbouring asymptomatic infections they represent reservoirs which guarantee the persistence of the parasite in the area.

Unité de Parasitologie expérimentale, Institut Pasteur, 75724 Paris Cedex 15, France.
Most of the mechanism involved in these evasion phenomena are not yet understood. However, research developed in many laboratories in the last ten years, has brought some light on the underlying molecular aspects of the parasite adaptation. These represent selected genetic mechanisms developed by the parasite in response to the host's immune response and/or, in some cases, a compromise between a parallel evolution of mechanisms developed by both, the parasite and the host.

In the present talk, we will analyse some of recent information obtained in the molecular analysis of Plasmodia's adaptation to the host's immune response.

\section{Antigenic variation}

Phenotypic antigenic variation has first been described in Plasmodium knowlesi (1) in relation to the SICA antigen (schizont-infected-cell-agglutination), located in the infected red cell (IRBC) membrane. Equivalent phenomena of antigenic variation have been described in experimental infection of Saimiri monkeys with Plasmodium falciparum, and in the infection of the natural primate host by $P$. fragile (reviewed in 2 and 3). In both cases, although the variant antigen could be detected by immunofluorescence in the IRBC membrane, it could not be identified. The intriguing observation of the role of spleen in the expression of the variant antigen is not yet understood. A parallel effect of 
the spleen has also been described in the expression of antigens implicated in sequestration in primate and rodent malaria (2, 4). P. falciparum antigens EMP-1(5) and/or Pf 11.1(6) could, in this case, be variant antigens, but no evidence has been obtained, to confirm this possibility. Furthermore, no information is available on possible molecular mechanisms responsable for antigenic variation in plasmodia. The existence of polygenic families coding for a variant antigen, like in trypanosome VSG seems unlikely. In contrast, the analysis of the molecular basis of variation of a merozoite-surface antigen of Plasmodium knowlesiP $140 \mathrm{Kd}$-observed under immune selective pressure, revealed extensive molecular and chromosome rearrangements together with point mutation or delections (7). In the squirrel monkey, we have recently obtained, by passive transfer of IgG immune, the selection of a $P$. falciparum variant which present molecular rearrangements apparently affecting more than one parasite antigen (Fandeur et al., in preparation).

\section{Allelic antigenic polymorphism}

Extensive polymorphism depending on variation of the amino-acid composition of some Plasmodium falciparum antigens has been described. The most studied cases of polymorphism are the $\mathrm{S}$ antigens and the merozoite surface antigens MSA-1 and MSA-2 (see 8 for review). S-antigens seem not to be responsible for the induction of protective immunity but the merozoite antigens, and MSA-1 in particular, are among the main candidates for vaccine preparations. MSA-1 is a proteolytic processing product of the major surface protein of the schizont-Pf 190. Extensive polymorphism was observed in the structure of the gene product. Comparative analysis of nucleotide sequences of the 190 genes cloned from different $P$. falciparum isolates defined 17 blocks that are conserved, semi-conserved and variable. A dimorphic model was proposed (9) to explain the extensive polymorphism of the protein as a result of intragenic recombination between two original alleles. This model, which defines the $\mathrm{K} 1$ and MAD20 P. falciparum strain as corresponding to parental allelic forms cannot, however, explain all the variation seen in MSA-1. As there is a variant (Ro-33) described as rare (10) and whose observed structure cannot be deduced from intergenic recombination of the $\mathrm{K} 1$ and MAD20 alleles. In a recent study in endemic area (11), we could demonstrate that this " rare " allele in fact represents a dominant variant among the isolates examined. The isolates were taken directly from patients without any in vitro culture.

\section{Molecular mimicry}

Micrimy has been long considered as a typical property of parasites in general and Plasmodia in particular (see 12 for review). However, its molecular basis started to become accessible to analysis only recently, when recombinant DNA techniques allowed the cloning of a large number of parasite genes and comparative analysis with host genes. This quickly demonstrated the existence of various degrees of homology between parasite gene products and host proteins. Two different situations must be, however, considered. First is the homology observed between enzymes and proteins which are supposed to have equivalent functions in both parasite and host. This is the case of aldolase (13) and the heatshock-protein family (14). The second situation is the homology observed between, on one hand, parasite protein antigens and, in the other hand, host proteins harbouring functions characteristic of higher organisms. This is the case of the asexual blood stage $P$. falciparum TRAP antigen (throbospondin anonimous related protein) (15) showing extensive homology with human's thrombospondin and properdin. Another astonishing homology was described between the Pf $25 \mathrm{~K}$ antigen of $P$. falciparum zygotes and ookynets with peptide domains of epidermal growth factor (16). In view of our ignorance on the functional role of the respective parasite proteins it is difficult to entirely exclude the possibility of common ancestor molecules which would explain the homology. It is, however, more tempting to think of a convergent molecular evolution which would be monitored by the selective advantage of molecular mimicry to evade recognition of parasite structures by the immune system. An intriguing homology was described in our laboratory between antigenic determinants present in a series of $P$. falciparum antigens of the Pf 11.1 family (Pf 11.1, Pf 332, RESA, Pf 102) and the thymic hormone thymosin (17). This raises the curious possibility that the parasite mimics immune modulators and directly participates in the (de)regulation of the host's immune response.

\section{Parasite interference with host immune response}

The ability of malarial parasites to induce an immune suppression in the host is frequently referred. No precise general mechanism has been, however, demonstrated for this suppression. In parallel, polyclonal $\mathbf{B}$ activation has also been frequently described in associated with malaria infection.

The presence of amino-acid sequences tandemly repeated in a large number of Plasmodia antigens has been demonstrated in the last few years. Cross-reactive dominant $B$ epitopes have been found between blocks of repeats present in the same parasite molecule or in different molecules, which create large networks of cross-reacting determinants. This particular structure has been considered as possibly associated with polyclonal B activation and hypergammaglobulinemia found in malaria. It could indirectly produce immunosuppression through a mechanism of limiting B-cell clonal expansion (including those with higher antigen affinity), because of competition between a too 
large number of activated B clones (18). Recent work in our laboratory supports this view and furthermore, indicates that cross-reactivity in Plasmodium falciparum antigens can also cover « self» antigenic determinants which introduce an additional element in the modulation of the host immune response (19). In another studies with the rodent malaria parasite Plasmodium chabaudi (20 and Ternynck et al., in preparation), we have shown the occurrence of $\mathrm{B}$ and $\mathrm{T}$ polyclonal activation during the acute infection and upon reinfection of sensitive or immune animals. An intense auto-antibody response was also observed against a panel of self antigens like actin, myosin, spectrin and tubulin. A substantial part of the induced autoantibodies seem to present a large polyspecificity. We have also obtained evidence that polyclonal activation in Plasmodium falciparum infections is dependent on antigenic mimicry rather than the presence of mytogenic products of the parasite. In the special case of heat-shock proteins, we could demonstrate that the parasite antigen is able to induce a self reacting response against the homologous human antigen (14).

\section{REFERENCES}

1. Brown K. N., Brown I. N. : Immunity to malaria. Nature, 1965, 208, 1286-1289.

2. Hommel M. : Antigenic variation in malaria parasite. Immunol. Today, 1985, 6, 28-33.

3. Hommel M., Semmof S.: Expression and function of eythrocyte-associated surface antigens in malaria. Biol. Cell, 1988, 64, 183-203.

4. Gilks C. F., Waliker D., Newbold C. I. : Relationship between sequestration, antigenic variation and chronic parasitism in Plasmodium chabaudi chabaudi, a rodent malaria model. Parasite Immunol., 1990, 12, 45-64.

5. Leech J. H. et al. : Identification of a strain specific malarial antigen exposed on the surface of $P$. falciparum infected erythrocytes. J. Exp. Med., 1984, 159, 1567-1575.

6. Scherf A. et al. : The 11.1 gene of P. falciparum codes for distinct fast evolving repeats. EMBO J., 1988, 7, 1129-1137.

7. Hudson D. E., Wellemms T. E., Miller L. H. : Molecular basis for mutation in a surface protein expressed by malaria parasites. J. Mol. Biol., 1988, 203, 707-714.

8. Anders R. F., Smythe J. A. : Polymorphic antigens in Plasmodium falciparum. Blood, 1989, 74, 1865-1875.

9. Mackay M. et al. : Polymorphism of the precursor for the major surface antigens of Plasmodium falciparum merozoites: studies at the genetic level. EMBO J., 1985, 4, 3823-3830.

10. Certa U. et al. : A naturally occurring gene encoding the major surface antigen precursor p190 of Plasmodium falciparum lacks tripeptide repeats. EMBO J., 1987, 6, 4137-4142.

11. Kimura E., Mattei D., Mana di Santi S., Scherf A. : Genetic diversity in the major merozoite surface antigen of Plasmodium falciparum: High prevalence of a third polymorphic form detected in strains dereived from malaria patients. Accepted in Gene.

12. Damian R. : Molecular mimicry revisited. Parasitol. Today, 1987, 3, 263-266.

13. Certa U., Ghersa P., Döbeli H., Matile H., Köcher H. P., Shrisvatava I. K., Shaw A. R., Perrin L. H. : Aldolase activity of a $P$. falciparum protein with protective properties. Science, 1988, 240, 1036-1038.

14. Mattei D., Scherf A., Bensaude O., Pereira da Silva L. : A heat schock-like protein of the malaria parasite Plasmodium falciparum induces autoantibodies. Eur. J. Immunol., 1989, 19, $1823-1828$.

15. Robson K. J. H. et al. : A highly conserved amino-acid sequence in thrombospondin, properdin and in proteins from sporozoites and blood stage of human malaria parasite. Nature, 1988, 335, 79-82.

16. Kaslow D. C. et al. : A vaccine candidate from the sexual stage of human malaria that contains EGF-like domains. Nature, 1988, 333, 74-77.

17. Dubois Ph. et al. : Structure and function of a thymic peptide is mimmicked by Plasmodium falciparum peptides. Ann. Institut Pasteur/Immunol., 1988, 139, 557-567.

18. Anders R. F. : Multiple cross reactivities among antigens of Plasmodium falciparum impair the development of protective immunity against malaria. Parasite Immunol., 1986, 8, 529-535.

19. Pereira da Silva L., Dubois P. : Possible role of amino-acid repeats in the evasion of Plasmodium falciparum from a host specific immune response. Molecular biology and infectious diseases. Elsevier, Paris, 1988, 223-232.

20. Falanga P. B., D'Imperio Lima M. R., Coutinho A., Pereira da Silva : Isotypic pattern of the polyclonal B cell response during primary infection by Plasmodium chabaudi and in immune protected mice. Eur. J. Immunol., 1987, 17, 599-603. 